\title{
On the best partitioning of the density functional energy
}

\author{
Mel Levy ${ }^{1}$ \\ Received: 28 August 2018 / Accepted: 20 September 2018 / Published online: 10 October 2018 \\ (C) Springer-Verlag GmbH Germany, part of Springer Nature 2018
}

\begin{abstract}
This essay discusses special features for two different ways of partitioning the density functional energy expression. The contribution, which is part of the special issue for Pratim Chattaraj, was stimulated by a thought-provoking suggestion by him at a recent conference.
\end{abstract}

Keywords Essay: density functional energy expression $\cdot$ Coordinate scaling $\cdot$ Constrained search

It is a pleasure to contribute this essay as part of the special issue for Pratim Chattaraj.

In density functional calculations, the ground-state electronic energy $E$ is traditionally partitioned as $E=\operatorname{Ven}[n]+\operatorname{Ts}[n]+$ $\mathrm{U}[\mathrm{n}]+\operatorname{Exc}[\mathrm{n}]$, where $\mathrm{n}$ is the ground-state electron density, Ven is the electron-nuclear attraction energy, Ts is the KohnSham non-interacting kinetic energy, $U$ is the classical Hartree energy, and Exc is the exchange-correlation energy. Alternatively, one could partition $E$ as $E=\operatorname{Ven}[n]+T[n]+$ Vee[n], where $T$ and Vee are, respectively, the true physical interacting kinetic and electron-electron repulsion energies.

Most often, Ts is computed from the optimum orbitals of the Kohn-Sham equations. However, for large complex molecular systems, there has been encouraging progress in approximating Ts directly as an orbital-free functional of $n$, and a lecture was given at the recent American Chemical Society meeting in New Orleans (March, 2018), where these orbitalfree advances were summarized.

During the discussion session, after the lecture, Pratim made an interesting and thought-provoking suggestion, whose essence I now recall: "Since $\mathrm{T}$ is the true kinetic energy, perhaps we should work on approximating an orbital-free $\mathrm{T}$ instead of Ts." The response was that with the use of Ts, we can directly take advantage of the known successful

This paper belongs to Topical Collection International Conference on Systems and Processes in Physics, Chemistry and Biology (ICSPPCB2018) in honor of Professor Pratim K. Chattaraj on his sixtieth birthday

Mel Levy

mlevy@tulane.edu

1 Department of Chemistry and Quantum Theory Group, Tulane University, New Orleans, LA 70118, USA approximations to Exc. Moreover, it is easy to make an approximation to Ts that satisfies its fundamental coordinate scaling requirement, which is that Ts scales homogeneously (as the square of the scale factor) when the coordinates of $n$ are scaled uniformly. The required scaling of $\mathrm{T}$ is more complicated because $\mathrm{T}=\mathrm{Ts}+\mathrm{Tc}$, where $\mathrm{Tc}$ is the positive kinetic contribution to the correlation energy.

Another consideration is the fact that $\mathrm{Ts}<\mathrm{T}$, and, independent of other factors, it is generally easier to approximate a smaller quantity than a larger one. Along these lines, the magnitude of Exc is less than the magnitude of Vee - U, which is approximated when one uses $\mathrm{T}$.

However, there is something enchanting about the possible use of the full T. Namely, the total energy, E (total), is of course given simply and elegantly by $\mathrm{E}($ total $)=-\mathrm{T}[\mathrm{n}]$ at the minimizing geometry. It is certainly worthwhile to think about this beautiful virial relation.

In closing, note that the constrained-search approach explains why Ts[n] scales homogeneously and why Ts[n] is less than T[n]. Specifically, observe that Ts [n] is the kinetic energy of the wavefunction that yields $\mathrm{n}$ and simultaneously minimizes just the kinetic energy expectation value, while $T[n]$ is the kinetic energy of the wavefunction that yields $n$ and minimizes the expectation value of the sum of the kinetic and electron-electron repulsion operators. 